\title{
Application of Information Management with Meeting Automation Tool
}

Andrey Naumenko, Alain Wegmann.

ICA, Swiss Federal Institute of Technology, Lausanne, Switzerland.

Phone: +41-21-693-2623, Fax: +41-21-693-6610.

E-mail: Andrey.Naumenko@epfl.ch; Alain.Wegmann@epfl.ch

\section{․ INTRODUCTION}

Presented work describes technologically supported solution that helps people in a workgroup to deal with information related to their common projects. The solution supports different scenarios of group organizations including the case of geographically distributed workgroups. It positions workgroup meetings as a key concept within a project framework. The paper will explain its foundations and show the positive value that it brings to everyday group work.

Diagrams 2-7 use UML [3].

\section{ORIGINS}

We present current results of "Meeting Automation Tool" (M.A.T.) research project that was initiated by Swisscom, the biggest Internet provider in Switzerland. They needed a solution to improve their regular project meetings automating standard processes such as production of meeting minutes document.

According to the client requirements, several constraints were established from the outset of the project. In particular:

- To use the tool, each of the meeting participants should have a PC or a laptop;

- The tool should support local and remote participations;

- $\quad$ M.A.T. should be easily used in combination with popular office software products;

- The tool should be designed to provide different levels of access to project-related information (see later in the Section 3.1). It was planned as a WWW based solution allowing functional integration with Personal Desktop Assistant (PDA) systems.

\section{a 3.SOLUTION}

M.A.T. provides a computer-supported framework for the meeting process that concentrates on capturing the content of meetings and its future use. We implemented strong points that were found in other solutions and proposed functionalities that we didn't find in existing products. For example, Ventana Groupsystems concentrates on meeting process and has nice utilities to support it, but it doesn't consider the evolution of the meeting-related information from one meeting to another. We can view meetings as milestones within projects, and the information discussed during a meeting can very well represent the current state of the project. So the evolution of this information has a value since it gives a continuous representation of the project. In our solution we have tried to provide a means to work with meeting-related information not only during a meeting but also before and after.

\subsection{Information Management foundations of the tool}

Model shown in Figure 1 was built to demonstrate theoretical foundations of the tool; it represents the domain of Information Management from the perspective of activities that can be performed in it. 


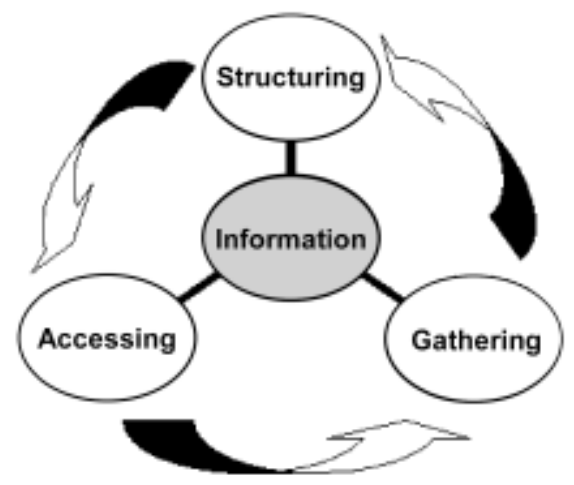

Figure 1. Information Management

The model shows a cycle of activities that are performed by collaborators in information-driven workgroups. Information itself is a central point of the model. People in everyday life are surrounded by numerous factors and events that offer an Access to information related to their ideas and activities. Every event, as soon as it can be considered relevant with regard to particular interests of a person or group, carries certain informational potential, that is to be consumed. Once a relevant piece of information has been obtained it needs to be combined with other pieces that are coherent with each other. Gathering of pieces combines together their informational potentials. It continues until the overall potential becomes significant enough to be realized by the person or group, in other words, for the idea that was behind the informational potential to be understood. Here, a physical analogy with Ohm's law may be very well applied: the bigger the resistance of a person or group against the realization of information, the bigger the informational potential is needed to generate the realization. As soon as an understanding of an idea is triggered, it needs to be saved for future applications. The Structuring of understanding (understanding being the way concepts are understood) can help in the storing the experience that is being accumulated. Structuring is just a convenient way to memorize what once was realized. The accumulated experience is then available for future access.

This model presents our vision of work with information in everyday life. The ideas highlighted for the Gathering part are exploration on experiential learning [1,2]. We designed our tool in correlation with the presented model. The core function of M.A.T. is to provide the means for the Gathering of information, more concretely, for collecting workgroup-related information through the evolution of a project. It is applied in workgroup meetings that are considered as milestones in a project timeline. The tool implements interfaces for Structuring and Accessing parts of the Information Management model. For Structuring it is linked with Group Information Base, which is designed to help people conceptualize information by means of Concept Maps [2]. It's shared within the group, and all information collected with M.A.T. goes there. For Accessing, the tool supports different levels of information access: Individual, Group and Community levels mapped to PDA, Intranet and Internet.

\subsection{Process and activities}

Figure 2 introduces a state diagram that represents a process supported by M.A.T. We can distinguish two parts: one of them is related to the project evolution, another to the meeting itself. The state diagram shows consecutive steps, which are performed in project, together with their stereotypes. The stereotypes represent sequence Information - Conceptualization - Action - Reflection, which is repeated iteratively in the process of project development. This sequence was taken from findings of Xavier Gilbert and to be published by Irwin Publishers: X. Gilbert, "Competing with Information”. A similar paradigm was expressed earlier by Jean Piaget $[4]$.

The state Organize Project represents part of the tool where a user can either organize a new project, including its name, description, participants and initial agenda, or change the organization of existing one. This state can be mapped to the Conceptualization part of the process. Projects can be considered as concepts, within a workgroup, that are either created or modified according to their previous evolution. 


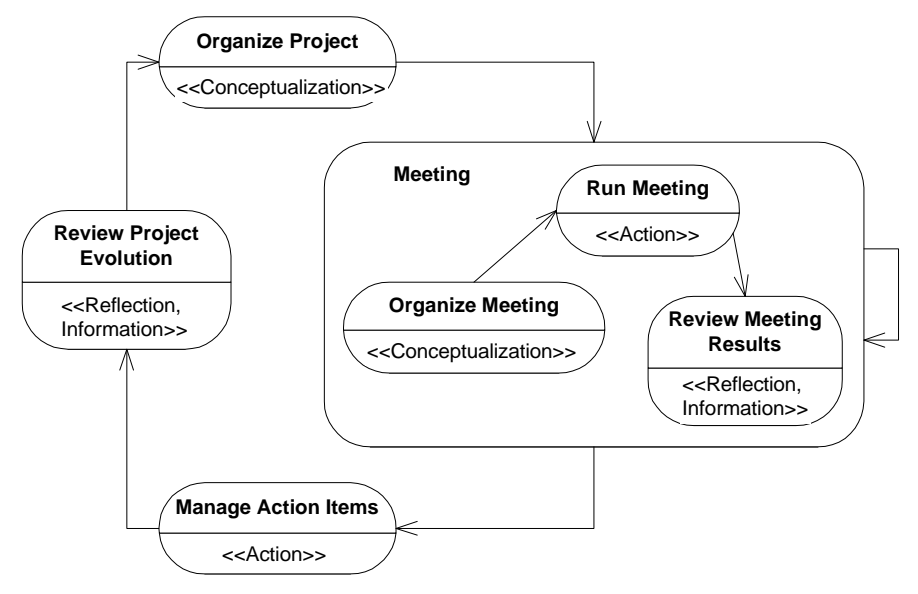

Figure 2. M.A.T. State Diagram

After conceptualization was performed, we can start acting on the concept that was developed. Here we move to project meetings that can be considered as a significant part of these actions. While being an action within a project, a meeting itself is a concept, and is separated in states within our tool. Organize Meeting, Run Meeting, and Review Meeting Results, - are the states that correspond to time phases: before, during and after a meeting. Meeting organization includes scheduling of meeting and its resources, creation of agenda, and sending invitations to participants. The running of a meeting implements collecting of the participants' contributions during a meeting and assigning action items within a project to corresponding responsible people. To review meeting results, users can read meeting minutes documents that are generated automatically after meetings, and review actions assigned during meetings. The last activity goes beyond the scope of the meeting concept. Manipulation with action items also contributes to a project lifecycle. An action is a concept by itself. It can be carried forward from one meeting to another; can have different statuses: not begun, completed, due, overdue; its statement can be modified over time. So in the tool we implemented a separate part, Manage Action Items, that supports all these aspects.

According to the described sequence of stereotypes, after making these different activities for a project, users pass to the Reflection and Information phases, which correspond to the Review Project Evolution state. Here the tool provides users with different views of project development over time. Dynamics of changes in a project's agenda, traces of project actions, and a timeline of key decisions may help to understand a current project's results and to make appropriate conclusions on its future development. The Group Information Base, which is complementary to the tool, should be also employed on this step, making use of the concepts that were contributed to it through a projects' evolution.

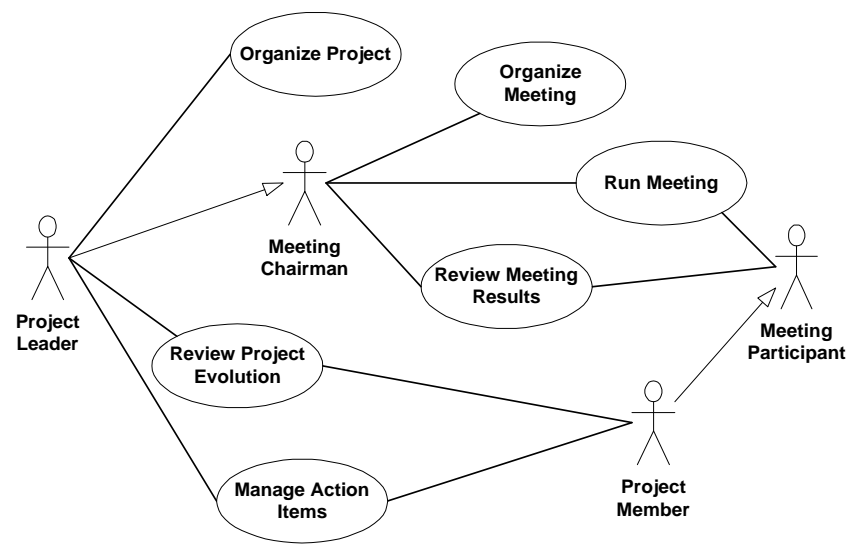

Figure 3. M.A.T. Use Case Diagram 
The tool implements Use Cases corresponding to the described states; they are shown on the Use Case diagram at Figure 3. Actors involved in the process are: Project Leader, Project Member, Meeting Chairman and Meeting Participant. Their relationships and actions with respect to the use cases are also shown on Figure 3.

Figure 4 presents meeting-related Use Cases from Figure 3 that consist of different activities that are performed before, during and after a meeting.

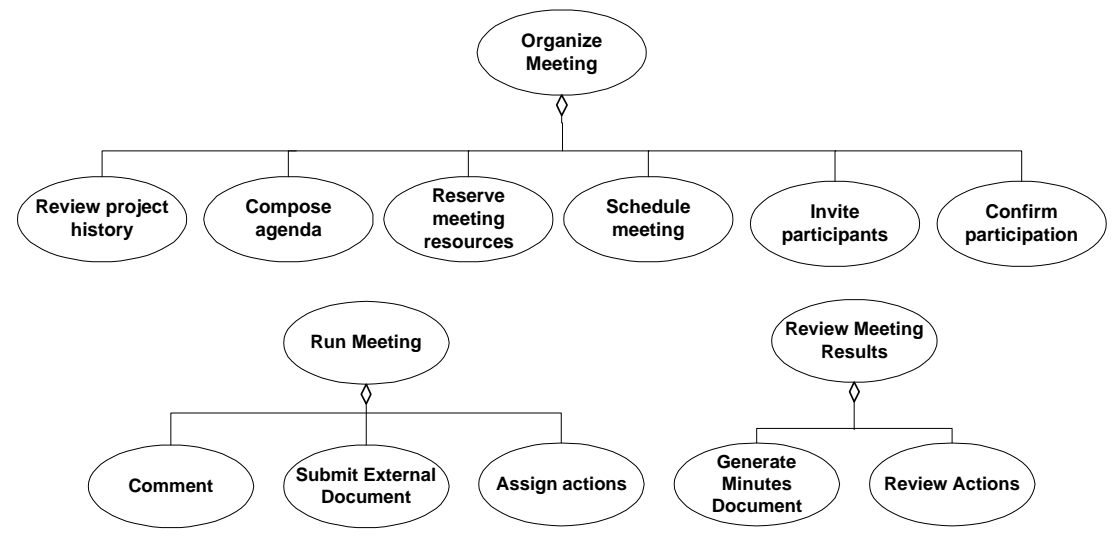

Figure 4. Implemented meeting-related Use Cases

\subsection{Structure and Functionality}

Figure 5 presents conceptual model implemented by M.A.T. Each Project has Project Participants and a set of Meetings. A meeting of a project has its Participants, who are at the same time participants of the project, and an Agenda that is produced taking into account the agenda, which is previous to it in the project. Agenda Items contribute to the meeting's agenda and consist of the Minutes and Actions that are entered during the meeting. The minutes themselves can be of different kinds, such as Comment, Question, Idea or Document.

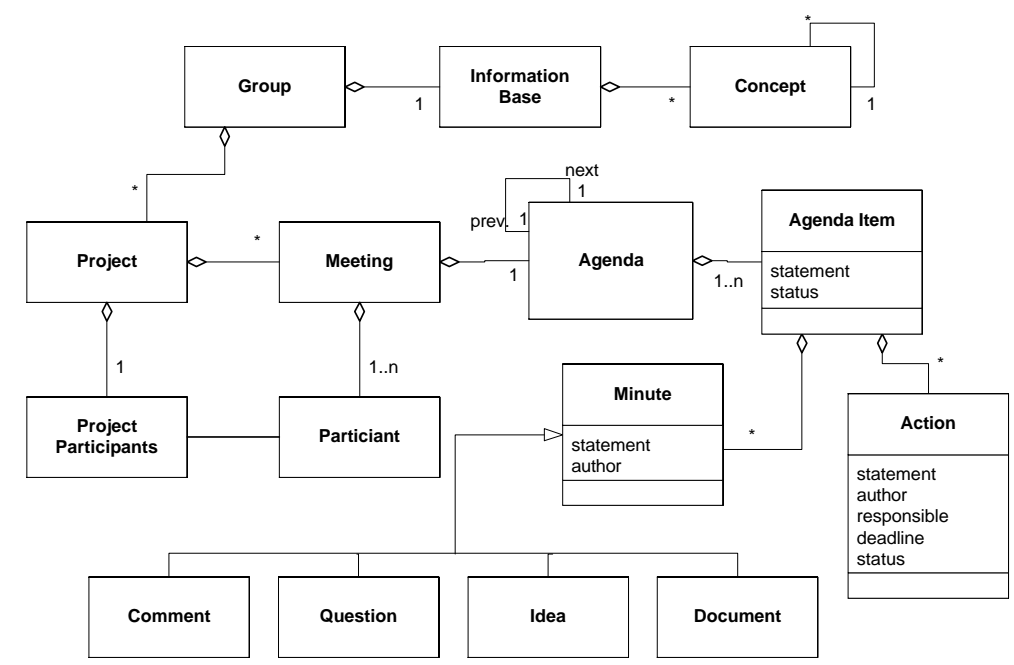

Figure 5. M.A.T. conceptual model 
Meeting participants use all the concepts that compose the concept of meeting. They can participate with their comments and decide to assign an action. All contributions during a meeting are authored by their corresponding contributors. An action has its responsible person, status, and, optionally, deadline.

\subsection{Architecture and Implementation}

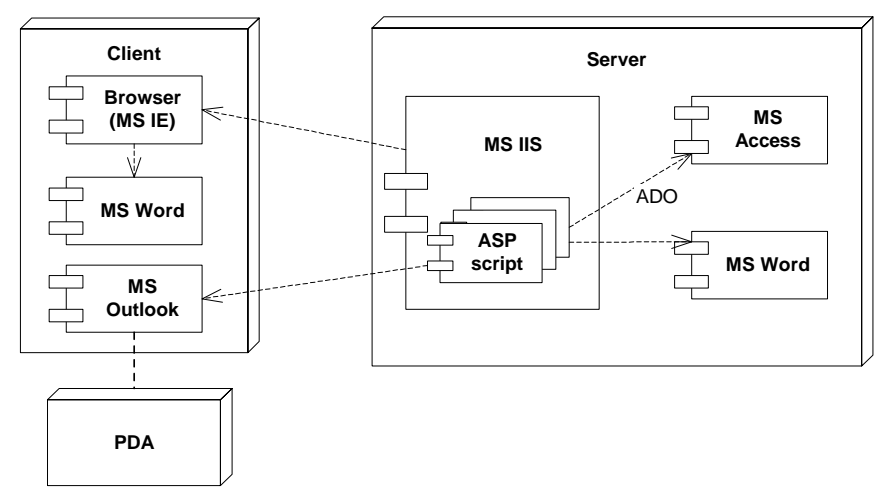

Figure 6. M.A.T. architecture

Keeping in mind the described use cases, the conceptual model and the technological constraints set from the client requirements, we built solution for the system architecture that is presented on Figure 6 .

The client part of the tool assumes that users have a WWW browser, installed on their PCs. By means of a browser via HTTP, clients access the main application based on a server that runs MS IIS as a WWW service.

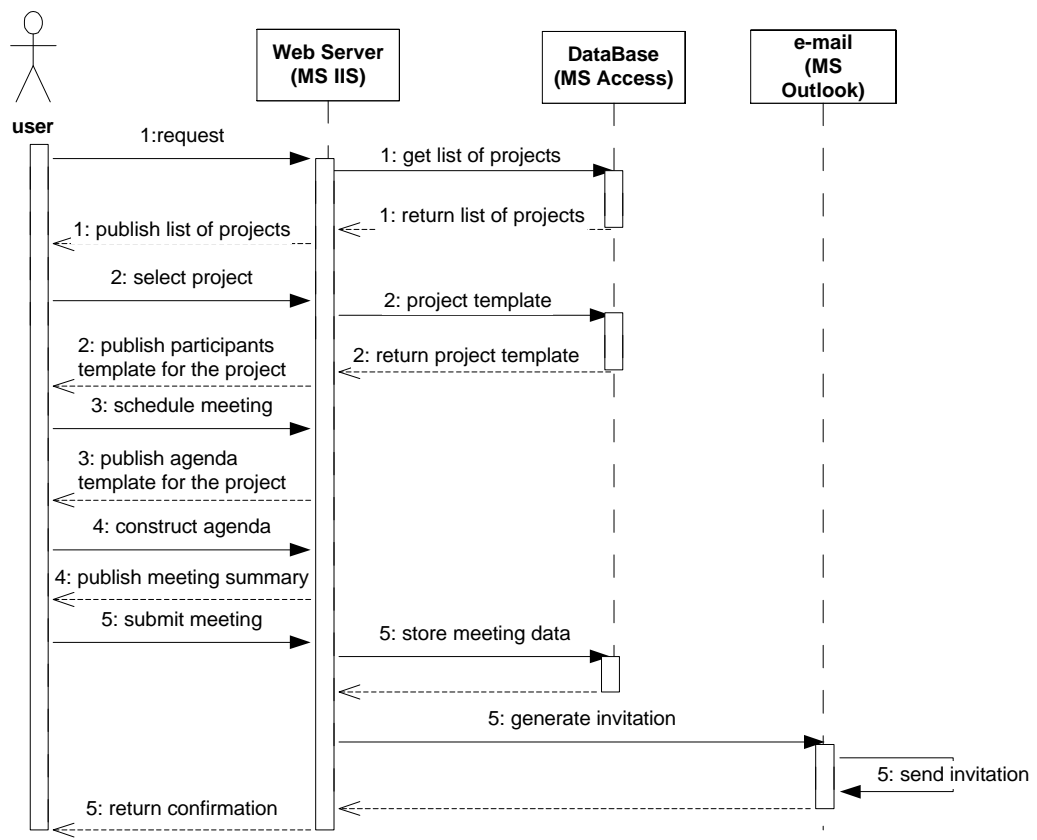

Figure 7. Scenario of organizing new meeting

The application consists of set of HTML pages with ASP scripts residing on them. These scripts are responsible for all use cases from Figure 3. Most of the scripts perform interface between users that interact with HTML 
pages and database that structures and stores meeting-related data. This interface is implemented with ADO; the database is done in MS Access. The automatic generation of meeting minutes, invitations and tasks messages are other applications of ASP scripts in our system. Integration of the tool with MS Word and MS Outlook was implemented here.

As an example Figure 7 shows sequence diagram for procedures that are performed when new meeting is organized.

\section{a 4. CONTRIBUTION}

M.A.T. solves problems of information gathering during workgroup meetings and offers a means for future management of captured information within the scope of the process that was described in Section 3.2. It also gives means to include project-related information in the Group Information Base for further conceptualization for exploration of given information as it was described in Section 3.1.

Architecture of the system allows integration of popular e-office software components and presents it on the WWW. It supports not only a web based (Internet) level of information access but also personal (mobile) access with the aid of PDA systems.

The system implements an interesting approach, presenting meetings that happen in everyday group work as milestones in the timeline of the projects that they contribute to. All data associated with these milestones contribute to the Group Information Base, represent evolution, informational concepts, and know-how of the project, and once collected can be applied as shared experiences in everyday group work.

The presented tool supports the process of a project's evolution in workgroups and contributes to Information Management for the project's content. The present version of M.A.T. is used for research and educational projects at EPFL-ICA and promoted for everyday use in research laboratory of Swisscom.

\section{口 REFERENCES}

[1]. Dewey J. (1938): 'Experience and Education', Simon \& Schuster, First Touchstone Edition 1997.

[2]. Novak J., (1998): 'Learning, Creating and Using Knowledge: Concept Maps as facilitative Tools in Schools and Corporations', Lawrence Erlbaum Associates Publishers 1998.

[3]. Object Management Group (1997): 'UML Specification', http://www.omg.org/techprocess/meetings/schedule/Technology_Adoptions.html\#tbl_UML_Specification

[4]. Piaget, J. (1970) : 'L'Epistémologie Génétique' Presses Universitaires de France, Que sais-je? collection, no. $1399 ; 1996$. 